\title{
Modelagem para recomendação de calagem e adubação para as principais regiões produtoras de grãos do Brasil na linguagem $\mathrm{SQL}$
}

Modeling for liming and fertilization recommendation for the main grain producing regions of Brazil in the SQL language

Henrique Alfonso Priebe ${ }^{1}$, Robert Rafael Araujo Oliveira ${ }^{2}$, Adriano Rodrigues Luz ${ }^{3}$, Eduardo Silveira Dornelles ${ }^{4}$, Gustavo Alves da Silva Bezerra ${ }^{5}$, Diones Vargas Dutra ${ }^{6}$, Vinícius Maran ${ }^{7}$ e Paulo Ademar Avelar Ferreira ${ }^{8}$

\section{1,2,3,4,5,6,7,8,Universidade Federal de Santa Maria, Cachoeira do Sul, Brasil}

henriquepriebe97@gmail.com; robertrafaelvg@gmail.com; adrianorodrigues329@gmail.com; eduardodornellessilveira@hotmail.com gustvoasbezerra@gmail.com; diones.de@redes.ufsm.br; vinicius.maran@ufsm.br; ferreira.aap@gmail.com

\section{Resumo}

Diante da importância do agronegócio para a economia brasileira, são necessárias tecnologias que busquem aumentar a eficiência do sistema produtivo, mas existem poucas ferramentas tecnológicas que realizam interpretações de análises de solo e recomendações de fertilizantes de forma eficiente e abrangente. Neste contexto, o sistema AgroFert propõe-se a realizar recomendações de corretivos e fertilizantes com base em análises de solo para diversas regiões e culturas de grãos do Brasil, através de dois sistemas: web e móvel, que utilizam uma base de conhecimento em manuais de recomendação de calagem e adubação. Portanto, este trabalho tem como objetivo apresentar o processo de modelagem conceitual e lógica de um banco de dados que possui regras de gerenciamento das informações nele contidas para interpretar os resultados de laudos de análise de solo e recomendar corretivos de solo e fertilizantes. Foram criadas regras que utilizam comparações, filtragens, operações matemáticas e operações lógicas, modeladas na linguagem SQL, e que quando executadas, geram interpretações de análises de solo e recomendações de corretivos e fertilizantes. O AgroFert abrange os estados Rio Grande do Sul, Santa Catarina, Paraná, Minas Gerais e São Paulo e o bioma do Cerrado. Além da modelagem conceitual, foram criadas 22 operações, totalizando aproximadamente 400 linhas de código, sendo possível recomendar fertilizantes para 13 culturas de grãos. Os resultados dos testes realizados demonstraram que a informatização dos dados presentes nos manuais do RS/SC e do Paraná facilitaram a interpretação dos dados presentes em laudos de análise de solo, além de aumentar a precisão e agilidade nos processos de recomendação de corretivos e fertilizantes pelos produtores.

Palavras-chave: Modelagem conceitual; SQL; Recomendação

\section{Abstract}

The agribusiness has a significant importance to the Brazilian economy. In this case, technologies are needed to increase the efficiency of the productive system, but there are few technological tools that perform interpretations of soil analysis and fertilizer recommendations efficiently and comprehensively. In this context, the AgroFert system proposes to make corrective and fertilizer recommendations based on soil analyzes for several regions and grain crops of Brazil, through two systems: web and mobile, which uses a knowledge base in liming and fertilization. Therefore, this work aims at presenting the conceptual and logical modeling process of a database that have rules of information management to interpret the results of soil analysis reports and to recommend soil correctives and fertilizers. Rules were created that use comparisons, filtering, mathematical operations and logical operations, modeled in the SQL language, and when executed, generate interpretations of soil analysis and recommendations for correctives and fertilizers. The AgroFert covers the states of Rio Grande do Sul, Santa Catarina, Paraná, Minas Gerais and São Paulo and the biome of Cerrado. In addition to the conceptual modeling, 22 operations were created, totaling approximately 400 lines of code, and it was possible to recommend fertilizers for 13 grain crops. The results of the tests showed that the computerization of the data present in the RS / SC and Paraná manuals facilitated the interpretation of the data present in soil analysis reports, besides increasing the precision and agility in the recommendations of corrective and fertilizer producers. 


\section{Introdução}

O agronegócio representou cerca de 23\% a 24\% do Produto Interno Bruto (PIB) do Brasil em 2017, de acordo com o IBGE (2017). Neste mesmo ano, o setor foi responsável por $48 \%$ de todas as exportações brasileiras (MINISTÉRIO DA AGRICULTURA, PECUÁRIA E ABASTECIMENTO, 2017). Isto evidencia a importância do agronegócio para a economia nacional. Para que este setor seja efetivo, assim como vários outros no país, ele depende de tecnologias que busquem aumentar a eficiência do sistema produtivo. Cerca de $50 \%$ dos aumentos de produtividade das culturas devem-se, dentre os diversos fatores de produção, ao manejo da fertilidade do solo por meio do uso eficiente de corretivos e fertilizantes (DJALMA et al., 2004). Desta forma, o uso de ferramentas que possibilitam melhor aproveitamento dos nutrientes pelas plantas também proporciona maior lucratividade aos produtores. Dentre essas ferramentas, a recomendação adequada de corretivos e nutrientes que atendam às necessidades do solo e das plantas torna-se fundamental para a sustentabilidade do sistema de produção e a sobrevivência do produtor, em virtude dos elevados custos de produção vinculados aos fertilizantes.

As recomendações de calagem e adubação para as diferentes culturas nos principais estados produtores do país fundamentam-se no conhecimento de especialistas, os quais desenvolvem tabelas de interpretação e recomendação de nitrogênio, fósforo e potássio, entre outros, nos diferentes manuais de recomendação publicados em alguns estados (Rio Grande do Sul, Santa Catarina, Paraná, São Paulo, Minas Gerais) e no bioma do cerrado. Estes manuais descrevem modelos matemáticos com sugestões de recomendação baseadas em diversos fatores químicos do solo e exigências nutricionais das culturas. No entanto, este processo se torna demorado, pois a interpretação e recomendação de corretivos e fertilizantes com base na consulta aos manuais é realizada de forma manual, bem como os cálculos baseados nas tabelas e modelos matemáticos. Além disso, os manuais sofrem atualizações, exigindo que o profissional tenha sempre em mãos o modelo mais recente publicado, para interpretações e recomendações mais precisas. Outra dificuldade é a variação do processo conforme o estado ou região, exigindo consultas ao manual do respectivo estado ou região onde a área de interesse se localiza.

Com base nisso, a informatização de dados existentes em livros ou artigos científicos publicados têm sido utilizada em diversos domínios de aplicação, de várias formas, para recomendar produtos, serviços ou processos para grupos específicos de usuários aumentando sua praticidade e eficiência (LU et al., 2015). No entanto, existem atualmente poucas ferramentas tecnológicas visando a interpretação de análise de solo e recomendação de corretivos e fertilizantes de forma eficiente para as principais culturas agrícolas e regiões produtoras, o que torna necessária sua realização ainda de forma manual (SANTOS et al., 2008; PAIVA DA SILVA et al., 2009).

Por isso metodologias e softwares de apoio têm sido desenvolvidos para auxiliar produtores na tomada de decisão referentes aos tipos e quantidades de insumos a serem aplicados em suas lavouras (COSTA, 2015; DA SILVA et al., 2015). Vários são os trabalhos na literatura que trazem algumas ferramentas de apoio à recomendação de calagem e adubação ao produtor (OLIVEIRA et al., 2005, SILVA et al., 2009, FERRERIA \& PINHEIRO, 2015, CARMONA et al., 2016 e OLEGO et al., 2014), mas estes sistemas e aplicativos são na maioria das vezes, limitados a poucas culturas e regiões.

A utilização de Modelos ou Sistemas tem se constituído numa estratégia eficiente e vantajosa para recomendar fertilizantes e corretivos (WITT et al., 1999; HAEFELE et al., 2003). Essa abordagem é recente no Brasil, e surgiu com o desenvolvimento do NUTRICALC (Sistema de Recomendação de Adubação para Eucalipto) (BARROS et al., 1995), sendo posteriormente aperfeiçoado e utilizado para diferentes grupos de culturas (TOMÉ JUNIOR et al., 2000; OLIVEIRA et al., 2005; SILVA et al., 2009). Além desses trabalhos, Silva (2012), desenvolveu um software para recomendação de calagem e adubação para o cultivo de tomateiros no estado de Minas Gerais em uma plataforma web. 
Carmona et al., (2016), por meio de programação em Delphi desenvolveram o programa chamado ADUBARROZ com o objetivo de manejar a adubação para a cultura do arroz irrigado no sul do Brasil. Oliveira et al., (2005) e Silva et al., (2009), modelando informações disponíveis na literatura sobre adubação e nutrição mineral da bananeira e do abacaxizeiro, desenvolveram o FERTCALC-BANANEIRA e o FERTCALC-ABACAXI. Estas plataformas apresentam a limitação de realizar recomendações de corretivos e fertilizantes para apenas uma cultura em uma determinada região do Brasil.

Diante disso, o objetivo deste trabalho é apresentar o processo de modelagem conceitual e lógica de um banco de dados utilizado por uma ferramenta alternativa para recomendação de corretivos e fertilizantes para as principais culturas de grãos das diferentes regiões produtoras do país. Denominada de AgroFert, foi elaborada por meio da sistematização e modelagem de informações disponíveis na literatura sobre adubação e nutrição mineral das culturas.

\section{Metodologia}

Nesta seção serão apresentados os passos realizados para a modelagem, implementação e integração do banco de dados proposto, com o sistema AgroFert.

\subsection{Desenvolvimento do Sistema AgroFert}

O AgroFert é um sistema de informação que está sendo desenvolvido com base nas informações existentes nos manuais de recomendação de calagem e adubação do bioma do cerrado, dos estados do Rio Grande do Sul/Santa Catarina, Minas Gerais, São Paulo e Paraná. O sistema foi estruturado de modo a permitir sua constante atualização à medida que novas informações e dados forem publicadas na literatura ou gerados pelos próprios usuários.

As recomendações de corretivos e fertilizantes a partir do AgroFert fundamentam-se nas informações contidas nos manuais das diferentes regiões do Brasil, baseadas nas tabelas de interpretações e nas tabelas de recomendação de nitrogênio, fosforo e potássio. Assim, este trabalho apresenta o processo de modelagem conceitual do banco de dados utilizado pelo sistema, bem como a modelagem das regras de interpretação e recomendação que são utilizadas pelo AgroFert. A modelagem realizada neste trabalho foi implementada no banco de dados utilizado pelo servidor de aplicação e no banco de dados utilizado pelo ambiente móvel.

\subsection{Modelagem Conceitual}

Inicialmente foram estudados os processos de adubação, calagem e aplicação de fertilizantes. Estes estudos realizados principalmente nos manuais de interpretação e recomendação foram necessários para a definição da taxonomia de conceitos relacionados aos processos. Com base nos estudos realizados, e na taxonomia obtida, modelou-se o banco de dados em formato relacional, para permitir consultas e operações de recomendação. Na Figura 1 é apresentado um diagrama entidaderelacionamento com alguns dos conceitos relacionados à recomendação.

Figura 1 - Diagrama Entidade-Relacionamento

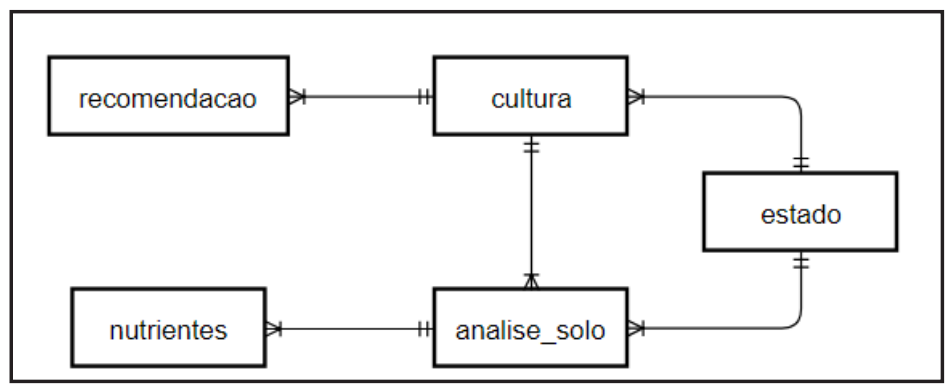


Através do diagrama é possível visualizar os parâmetros que exercem influência direta no resultado recomendação. Assim como nos manuais, a quantidade de fertilizante varia de acordo com a cultura, com o resultado da análise de solo e de acordo com os nutrientes necessários determinados através da interpretação da análise. Também influencia a recomendação, o estado ou região onde a área de interesse se localiza, pois determina à qual manual estarão ligadas as informações utilizadas.

A criação, conforme o diagrama entidade-relacionamento, das entidades, atributos e relacionamentos do banco de dados, bem como a criação dos processos em SQL, foram realizados através da ferramenta de edição e desenvolvimento $M y S Q L$ Workbench Visual Database Designer na versão 6.3.10, conhecida também como um Sistema de Gerenciamento de Banco de Dados (SGBD).

\subsection{Modelagem Lógica}

As interpretações das análises de solo tratam-se de classificações de cada nutriente contido na amostra de solo recolhida, em cinco níveis: muito baixo, baixo, médio, alto e muito alto. Os manuais de recomendação de calagem e adubação apresentam tabelas com limites inferiores e superiores para classificação de cada nutriente conforme estes níveis, ou seja, o profissional da área identifica a faixa de valores dentro da qual cada nutriente se encontra, e com isso o classifica quanto ao nível. É justamente esta etapa do processo que as consultas de interpretação automatizam, pois os limites contidos nos manuais foram transcritos para tabelas do banco de dados. Além disso, os resultados da análise de solo digitados pelo usuário na interface de um dos sistemas também são devidamente armazenados em uma tabela. Portanto, cada consulta de interpretação modelada em SQL é responsável por comparar a quantidade do nutriente contido na amostra de solo com os limites contidos na respectiva tabela, e retornar um número de 1 a 5 , correspondente ao nível de classificação, sendo 1 equivalente a "muito baixo" e 5 a "muito alto".

As recomendações de fertilizantes também são realizadas com base em tabelas dos manuais, que contém a quantidade de cada nutriente (em kg/ha) a ser aplicada de acordo com o respectivo nível determinado na interpretação, bem como o adicional a ser somado, referente à expectativa de produtividade desejada pelo usuário. De maneira geral, esses são os dois critérios levados em consideração para recomendação de fertilizantes. Porém, cada nutriente possui particularidades que envolvem outras variáveis, tais como cultura anterior, sequência de cultivos, tipo de sistema, etc. Assim como para as interpretações, as informações referentes à recomendação de nutrientes também foram inseridas em tabelas específicas do banco de dados.

Os processos de comparação da interpretação de cada nutriente com as interpretações pré-definidas nas respectivas tabelas, bem como a soma referente ao adicional da expectativa de produtividade, foram automatizados pelas consultas de recomendação modeladas em SQL. À primeira vista este parece ser um processo simples de automatização. Mas, como referido anteriormente, cada nutriente possui particularidades, envolvendo mais variáveis e condições específicas, aumentando o número de linhas de código em SQL em relação às consultas de interpretação, e consequentemente, à dificuldade em se obter consultas totalmente funcionais.

\section{Avaliação e resultados}

A interface web do AgroFert foi desenvolvida visando facilitar a operação por parte do usuário, com cada recurso podendo ser acessado e utilizado de maneira intuitiva. Além disso, a página inicial exibida ao acessar o sistema (Figura 2), traz uma sequência de etapas detalhadas em vídeos tutoriais, com o objetivo de auxiliar o usuário na utilização da ferramenta. 
Figura 2 - Página inicial AgroFert

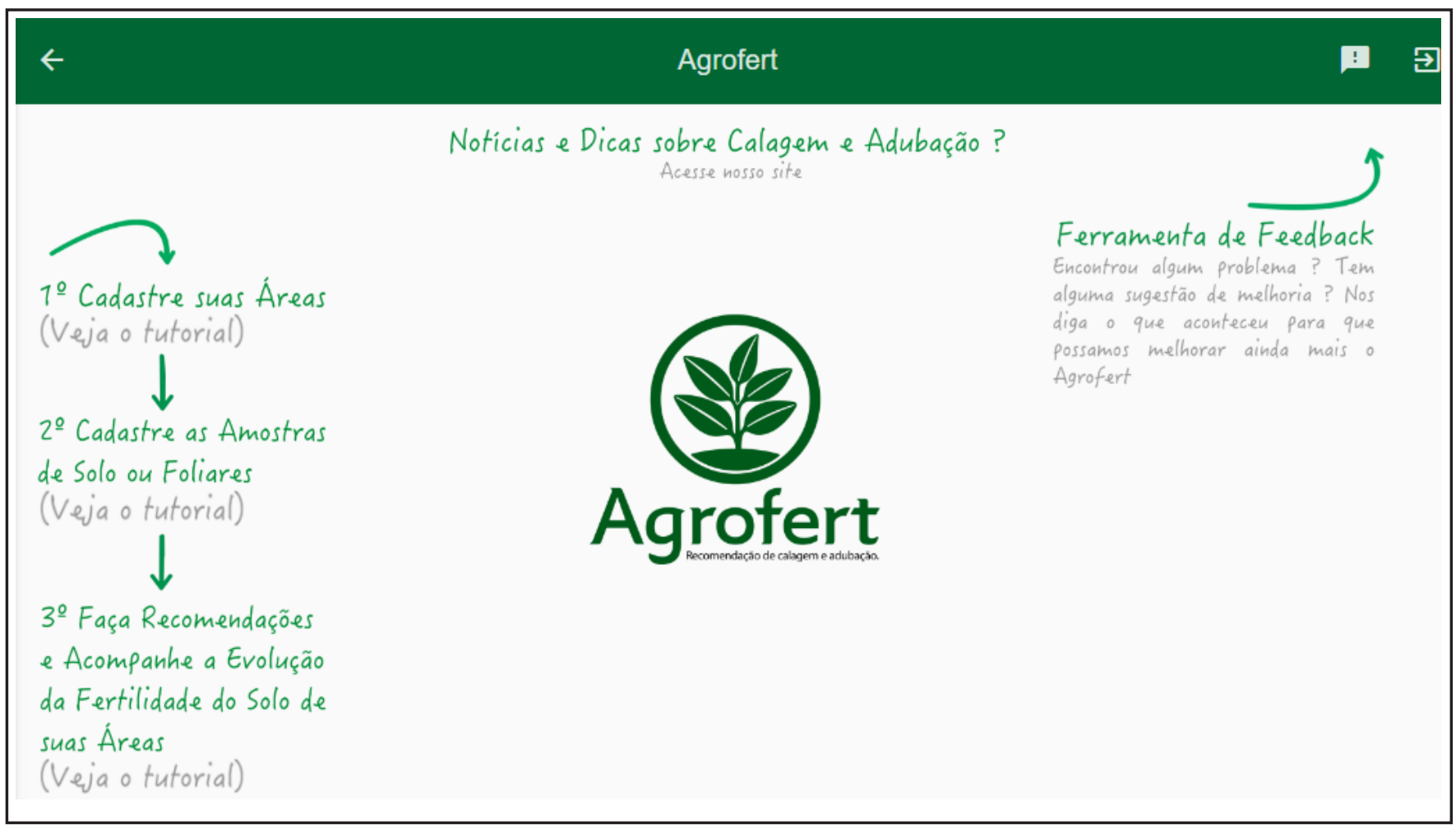

Além da sequência de etapas, há na parte superior da página inicial um link de acesso ao site AgroFert, onde encontram-se notícias sobre calagem, adubação e temas relacionados à recomendação de fertilizantes. No canto superior esquerdo da página está destacado o botão de Feedback, que permite ao usuário relatar problemas e sugestões à equipe de desenvolvimento do sistema.

Após cadastrar uma área e uma amostra de solo ou foliar, já é possível realizar interpretações e recomendações, obtendo resultados após a inserção de algumas informações adicionais, como apresentado na Figura 3. Na ocasião, foi realizada uma recomendação para a cultura da soja, com expectativa de produtividade de 3,6 toneladas por hectare, estando a área localizada no Rio Grande do Sul.

Os resultados de interpretação e recomendação apresentados na interface são provenientes da execução de consultas SQL, com as quais, desde a concepção, foram executados testes parciais, comparando sempre os resultados mostrados por elas com os obtidos consultando os respectivos manuais. Após correta correspondência nos testes parciais, as consultas foram implementadas nos sistemas web e móvel, iniciando-se a fase de calibração, onde vários testes de interpretação e recomendação sob diferentes condições foram realizados e novamente comparados com os resultados obtidos pelo método tradicional. 
Figura 3 - Realização de interpretação e recomendação

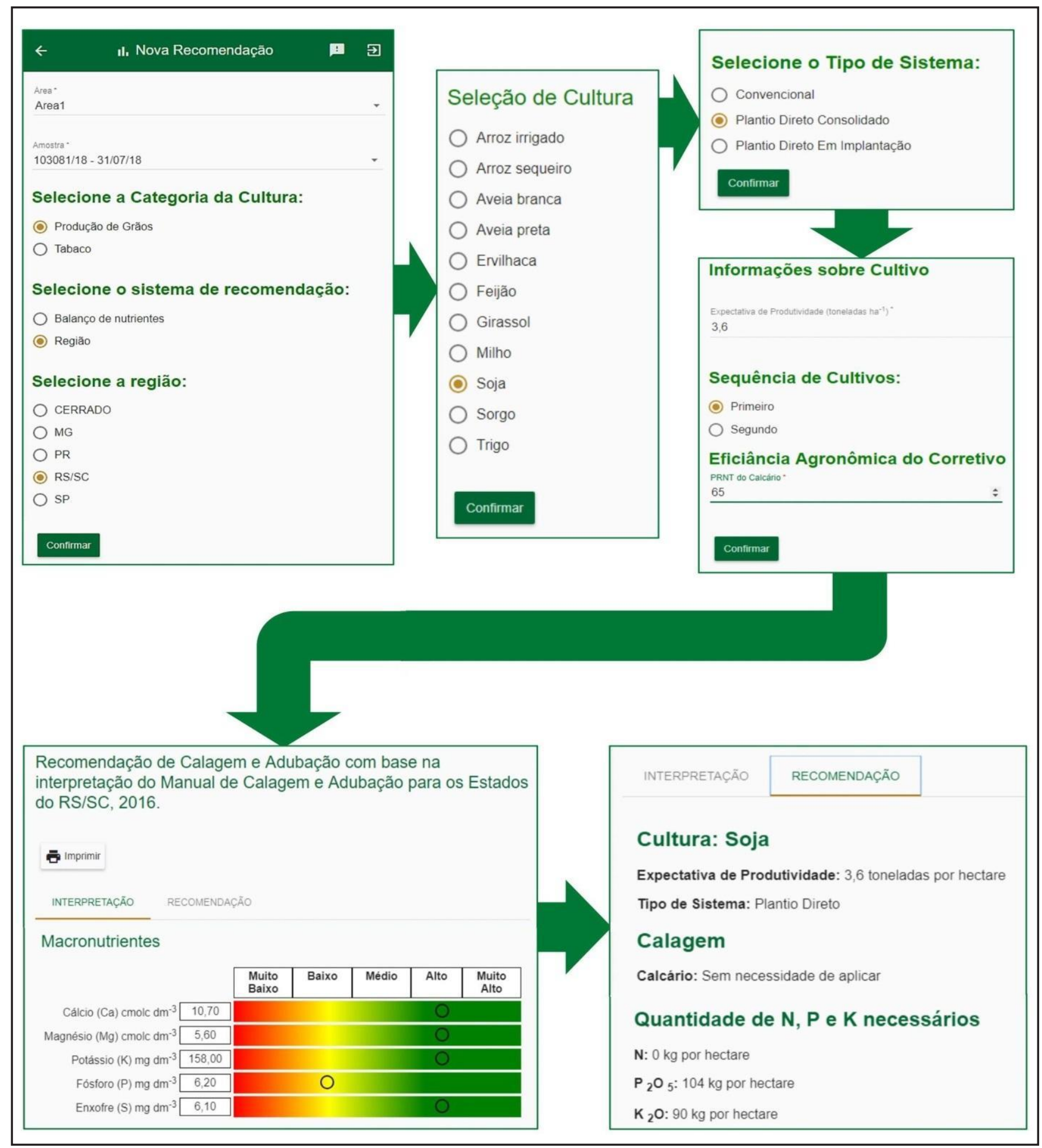

Na Tabela 1 é apresentada uma comparação entre os resultados obtidos pelo AgroFert e pelo método tradicional consultando o manual de recomendação do Rio Grande do Sul, para a recomendação de corretivos e fertilizantes para a soja. Tanto pelo AgroFert quanto pelo método tradicional, os resultados encontrados para recomendação de corretivos e fertilizantes foram os mesmos. 
Tabela 1 - Recomendação de corretivo e fertilizantes para a cultura da soja para o estado do Rio Grande do Sul comparando o sistema AgroFert e o método tradicional

\begin{tabular}{lll}
\hline Nutriente & Recomendação AgroFert & Recomendação método tradicional \\
\hline Corretivo & 3,6 toneladas por hectare & 3,6 toneladas por hectare \\
\hline $\mathbf{N}$ & $0 \mathrm{~kg}$ por hectare & $0 \mathrm{~kg}$ por hectare \\
$\mathbf{P}_{2} \mathbf{O}_{\mathbf{5}}$ & $104 \mathrm{~kg}$ por hectare & $104 \mathrm{~kg}$ por hectare \\
$\mathbf{K}_{\mathbf{2}} \mathbf{O}$ & $90 \mathrm{~kg}$ por hectare & $90 \mathrm{~kg}$ por hectare \\
\hline $\mathbf{S}+$ Micro & Sem necessidade de aplicar & Sem necessidade de aplicar \\
\hline
\end{tabular}

Outro teste realizado foi o de tempo para recomendações utilizando o AgroFert, e o método tradicional (Manual). Para realizar este teste, cinco especialistas efetuaram recomendações utilizando uma amostra de solo e todas as culturas de grãos presentes em cada estado. Foi realizada uma comparação entre o AgroFert e o método tradicional de recomendação, do tempo médio utilizado para as recomendações de corretivos e fertilizantes (Figura 4). Foram avaliados o tempo necessário para realizar a recomendação de corretivos, gesso agrícola (exceto para o Rio Grande do Sul e Santa Catarina) e fertilizantes (NPK $+\mathrm{S}+$ Micronutrientes), para todas as culturas de grãos de cada região do sistema AgroFert. Para o bioma do cerrado o tempo médio gasto para realizar as recomendações de corretivos, gesso agrícola e fertilizantes $(5$ formulações de NPK $+\mathrm{S}+$ Micronutrientes) foi de 4,22 minutos pelo AgroFert, e de 15,4 minutos pelo método tradicional (Figura 4).

Figura 4 - Tempo gasto para recomendar corretivos, gesso agrícola e fertilizantes ( 5 formulações de NPK $+\mathrm{S}+$ Micronutrientes) utilizando o AgroFert e o método tradicional (Manual) de recomendação
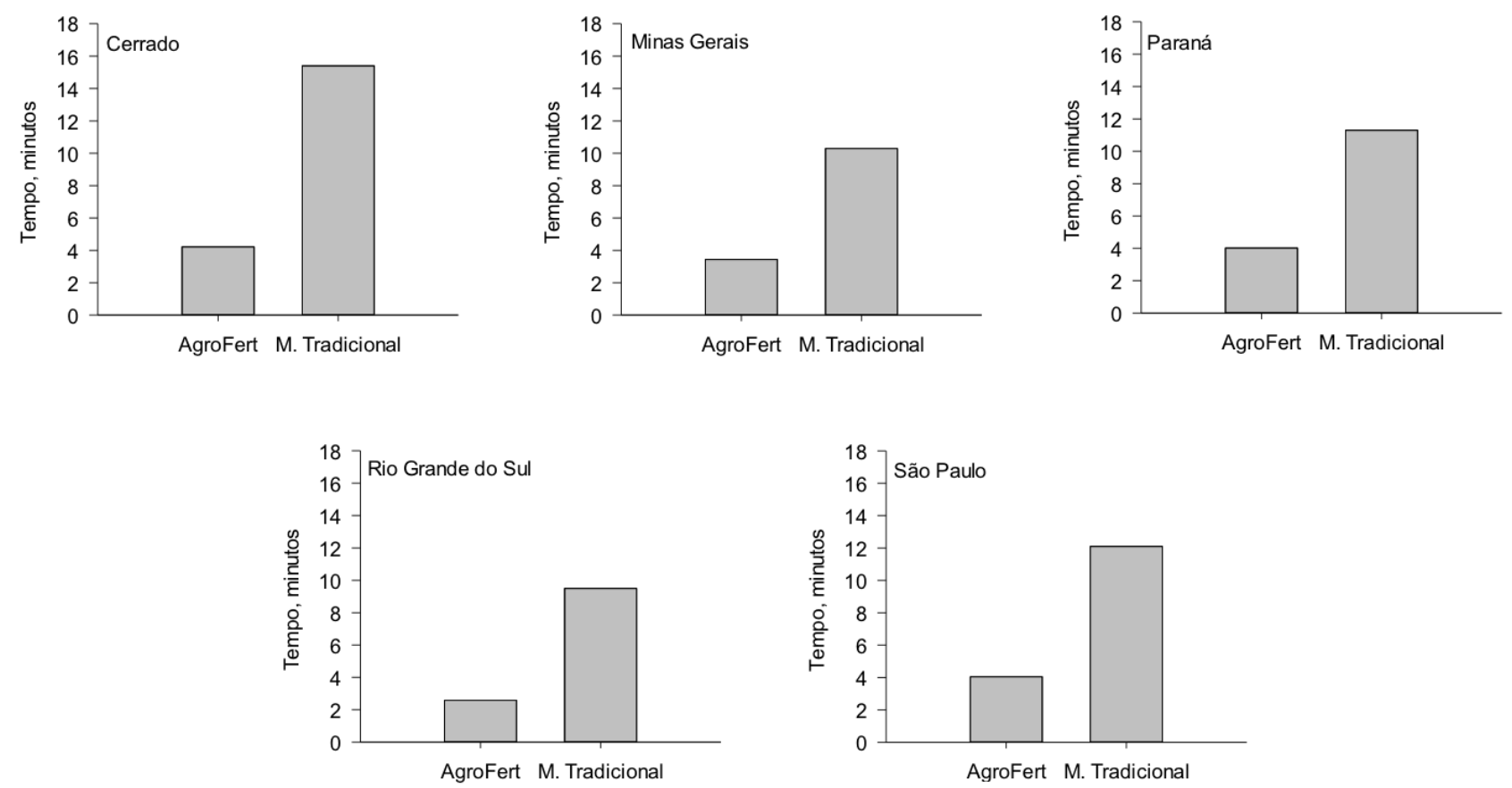
Para a região de Minas Gerais, Paraná e São Paulo as recomendações de corretivos, gesso agrícola e fertilizantes (5 formulações de NPK $+\mathrm{S}+$ Micronutrientes), utilizando o AgroFert foram de 3,43, 4,01 e 4,08 minutos, e pelo método tradicional de 10,29, 11,30 e 12,10 minutos, respectivamente. As recomendações de corretivos e fertilizantes (5 formulações de $\mathrm{NPK}+\mathrm{S}+$ Micronutrientes) realizadas pelo método tradicional para o Rio Grande do Sul levaram 9,50 minutos, e utilizando o AgroFert de 2,58 minutos. Em relação aos diferentes trabalhos de softwares que encontram-se publicados na literatura e disponíveis (TOMÉ JUNIOR et al., 2000; OLIVEIRA et al., 2005; PAIVA DA SILVA et al., 2009; SILVA 2012; CARMONA et al., 2016), o AgroFert é um sistema mais completo devido a sua maior abrangência de regiões, e a um número superior de culturas cadastradas em seu banco de dados.

\section{Conclusões}

Diante do exposto, percebe-se que é possível criar consultas em linguagem SQL para interpretar análises de solo e recomendar corretivos e fertilizantes, desde que as informações contidas nos manuais de recomendação sejam modeladas adequadamente. Além disso, as consultas são responsáveis por todas as operações lógicas e matemáticas que envolvem o processo de recomendação, o que facilita e agiliza o tratamento de erros. Elas também são facilmente integráveis ao sistema web, pois este é responsável pelo gerenciamento, apenas executando-as em ordem correta.

A ferramenta AgroFert abrange todas as culturas de grãos de maior interesse econômico dos estados do Rio Grande do Sul, Santa Catarina, Paraná, Minas Gerais, São Paulo e do bioma cerrado. O sistema recomenda corretivos e fertilizantes de forma precisa e ágil, facilitando a vida do produtor rural. Os próximos passos visam à inclusão de novas culturas no sistema, bem como a criação de novas consultas de interpretação e recomendação, para tornar o AgroFert ainda mais completo.

\section{Agradecimentos}

Os autores agradecem ao CNPq pela concessão de bolsa de IC no programa PIBIC/CNPq e à FAPERGS.

\section{Referências}

BROCH L D, SIDNEI K R. Fertilidade do solo, adubação e nutrição da cultura do Milho. 2011-2012; Tecnologia e produção: Soja e Milho.

CAHN M, HARTZ T, SMITH R, NOEL B, JOHNSON L, MELTON F. [CropManage: an online decision support tool for irrigation and nutrient management]. Proceedings of the Western Nutrient Management Conference. Rev. Eletr. Enf. [Internet] 2015 March [cited 2018 feb 17]. Aviable from: <http://www.ipni.net/ipniweb/conference/wnmc.nsf/e0f 085ed5f091b1b852579000057902e/4be3031d1d87927a85257e37004fa7a8/\$FILE/WNMC2015\%20Cahn\%20pg9.pdf .

CARMONA F C, ANGHINONI I, MARTINS A P, BOENI M. ADUBARROZ: uma experiência brasileira em recomendações de adubação e calagem para o arroz irrigado via sistema computacional. Rev. Eletr. Enf. [Internet] 2016 [cited 2018 feb 03]; 46(11):1959-1962. Aviable from: http://revistas.bvs-vet.org.br/crural/article/view/33353.

COSTA M M. Recomendação de adubação em agricultura de precisão com base no modelo CERES-Maize. 2015.

DA SILVA S V, TUCCI C A F, SANTOS J Z L. Calagem e adubação com potássio, boro e cobre em laranjeira 'pêra' em Manaus-AM. 2015. 
DE OLIVEIRA F H T, NOVAIS R F. Desenvolvimento de um sistema para recomendação de adubação para a cultura da bananeira. Revista Brasileira de Ciência do Solo. 2005;29(1):131-143.

FERREIRA JUNIOR L D, PINHEIRO M C. Desenvolvimento de software de gestão da produção cafeeira: módulo de prescrição e planejamento da adubação. 2015.

INSTITUTO BRASILEIRO DE GEOGRAFIA E ESTATÍSTICA [Internet]. Brasília: Ministério da Agricultura, Pecuária e Abastecimento (BR) [cited 2018 aug 27]. Aviable from: http://www.agricultura.gov.br/noticias/agropecuaria-puxa-o-pib-de2017.

LU J, WU D, MAO M, WANG W, ZHANG G. [Recommender system application developments: a survey]. Decision Support Systems. 2015;74:12-32.

MINISTÉRIO DA AGRICULTURA, PECUÁRIA E ABASTECIMENTO. Ministro da agricultura fala sobre a forca do agronegócio em seminário em brasilia; 2017 Mai 08 [cited 2018 aug 27]. Aviable from: http://www.agricultura.gov.br/noticias/ministro-da-agricultura-fala-sobre-a-forca-do-agronegocio-em-seminario-em-brasilia.

OLEGO M A, Paz J M, Visconti F, Garzón J E. [Predictive modelling of soil aluminium saturation as a basis for liming recommendations in vineyard acid soils under Mediterranean conditions]. Soil science and plant nutrition. 2014;60(5):695-707.

PAIVA DA SILVA A, Alvarez V H V, Souza A P, Neves J C L, Novais R F, Dantas J P. Sistema para recomendação de fertilizantes e corretivos para a cultura do abacaxi - FERTICALC-ABACAXI. Revista Brasileira de Ciência do Solo. 2009;33:1269-1280.

SANTOS F C, NEVES J C L, NOVAIS R F, ALAREZ V H V, SEDIYAMA C S. Modelagem da recomendação de corretivos e fertilizantes para a cultura da soja. Revista Brasileira de Ciência do Solo. 2008;32:1661-1674.

SILVA T M D. Software for recommendation of liming and fertilization of tomato plants in the state of minas gerais. [dissertation]. Alfenas: Mestrado em Ciência Agrárias/Universidade Jose do Rosario Vellano; 2012.

WENDLING A, ELTS F L F, CUBILLA M M, AMADO T J C, MIELNICZUK J, LOVATO T. Recomendação de adubação nitrogenada para trigo em sucessão ao milho e soja sob sistema plantio direto no Paraguai. Revista Brasileira de Ciência do Solo. 2007;31(5): 985-994. 